\title{
ASTEROSEISMIC ANALYSIS OF THE PRE-MAIN-SEQUENCE STARS IN NGC 2264
}

\author{
D. B. Guenther ${ }^{1}$, T. Kallinger ${ }^{2}$, K. Zwintz 2 , W. W. Weiss ${ }^{2}$, R. Kuschnig ${ }^{2}$, M. P. Casey ${ }^{1}$, J. M. Matthews ${ }^{3}$, \\ A. F. J. Moffat ${ }^{4}$, S. M. Rucinski ${ }^{5}$, D. SASSElov ${ }^{6}$, And G. A. H. WALKeR ${ }^{3}$ \\ ${ }^{1}$ Department of Astronomy and Physics, Saint Mary's University, Halifax, N.S., B3H 3C3, Canada \\ 2 Institut für Astronomie, Universität Wien, Türkenschanzstrasse 17, A-1180 Vienna, Austria \\ ${ }^{3}$ Department of Physics and Astronomy, University of British Columbia, 6224 Agricultural Road, Vancouver, BC V6T 1Z1, Canada \\ ${ }^{4}$ Observatoire Astronomique du Mont Mégantic, Départment de Physique, Université de Montréal C. P. 6128, Succursale: Centre-Ville, Montréal, QC H3C 3J7, \\ Canada \\ 5 Department of Astronomy and Astrophysics, University of Toronto, Toronto, ON M5S 3H4, Canada \\ ${ }^{6}$ Harvard-Smithsonian Center for Astrophysics, 60 Garden Street, Cambridge, MA 102138, USA \\ Received 2009 July 14; accepted 2009 September 14; published 2009 October 6
}

\begin{abstract}
NGC 2264 is a young open cluster lying above the Galactic plane in which six variable stars have previously been identified as possible pre-main-sequence (PMS) pulsators. Their oscillation spectra are relatively sparse with each star having from 2 to 12 unambiguous frequency identifications based on Microvariability and Oscillations of Stars satellite and multi-site ground-based photometry. We describe our efforts to find classical PMS stellar models (i.e., models evolved from the Hayashi track) whose oscillation spectra match the observed frequencies. We find model eigenspectra that match the observed frequencies and are consistent with the stars' locations in the HR diagram for the three faintest of the six stars. Not all the frequencies found in spectra of the three brightest stars can be matched to classical PMS model spectra possibly because of effects not included in our PMS models such as chemical and angular momentum stratification in the outer layers of the star. All the oscillation spectra contain both radial and nonradial $p$-modes. We argue that the PMS pulsating stars divide into two groups depending on whether or not they have undergone complete mixing (i.e., have gone through a Hayashi phase). Lower mass stars that do evolve through a Hayashi phase have oscillation spectra predicted by classical PMS models, whereas more massive stars that do not, retain mass infall effects in their surface layers and are not well modeled by classical PMS models.
\end{abstract}

Key words: stars: evolution - stars: pre-main sequence - stars: oscillations - open clusters and associations: individual (NGC 2264)

\section{INTRODUCTION}

Following Hayashi (1961) and Iben (1965) the standard picture of pre-main-sequence (PMS) evolution begins on the Hayashi track, a nearly constant temperature line in the cool region of the HR diagram, where stars are forced (by the surface boundary condition) to be fully convective. The PMS star evolves down the Hayashi track, and then, because its core heats up due to gravitational contraction, the interior opacities drop, convection recedes to the outer layers, and the PMS star evolves toward hotter regions of the HR diagram. Eventually, the interior temperatures are high enough to initiate hydrogen burning in the core. Energy from hydrogen fusion quickly increases until it replaces gravitational energy as the dominant energy source. Since the movement of the star in the HR diagram, at this point, slows from a thermodynamic timescale (radiated power derived from gravitational collapse) to a nuclear timescale (radiated power derived from hydrogen burning), stars of different masses bunch up along a line of points running diagonally from high luminosity and high temperature to low luminosity and low temperature in the HR diagram. This curve roughly defines the zero-age main sequence (ZAMS). Classical PMS models are chemically homogeneous, since they have evolved from a fully convective phase on the Hayashi track and have not yet initiated core hydrogen burning (Iben 1965).

Stahler et al. (1980a, 1980b, 1981), Stahler (1983) and later, for models relevant to this study, Palla \& Stahler (1992, 1993; hereafter referred to collectively as Palla \& Stahler) are responsible for a significant improvement to the modeling of PMS stellar evolution. In classical PMS modeling, the environment within which the stars are formed is ignored. But stars do not begin their evolution in pristine environments. They are surrounded by a disk of primordial matter that, during the star's early life (protostar stage), accretes onto the star. This process continues until some mechanism, not yet fully understood, halts the flow of matter onto the surface of the star, and blows away the surrounding obscuring dust and gas. Palla \& Stahler (and their collaborators Shu \& Tamm) investigated the effects of this more realistic picture by first modeling the hydrodynamics of the surrounding shells of material (Stahler et al. 1980a, 1980b, 1981), obtaining results that improve on the first hydrodynamical models of PMS stars by Larson (1972). They then modified their PMS stellar evolution modeling to include the mass infall boundary conditions obtained from their hydrodynamic simulations. The mass of a star, then, slowly builds up over time until the radiative luminosity is strong enough to halt the mass infall. Because the matter falling onto the star carries deuterium, deuterium burning continues much longer than in classical PMS modeling. This leads to most of the structural differences between the Palla \& Stahler models and classical PMS models. The point where infall ceases is called the birthline (Stahler 1983) since this is where the star emerges from its parent molecular cloud and becomes visible as a star-like object. Above the birthline, mass infall is still taking place hidden from view behind layers of gas and dust. The existence and approximate location of the birthline is confirmed by observations of young clusters with the predicted birthline for a mass infall rate of $\sim 10^{-5} M_{\odot} \mathrm{yr}^{-1}$ matching the observed color-magnitude diagrams of the youngest open clusters, e.g., the Orion nebula cluster (Palla \& Stahler 1999). 
The Palla \& Stahler models show (similar to Larson 1972) that stars with masses greater than 2.5-3.0 $M_{\odot}$ do not evolve through a Hayashi phase, but exit directly into the radiative PMS phase. Without going through complete mixing, and with lower entropy and radii than predicted by classical PMS evolutionary models, the surface layers of these PMS stars differ from predictions of classical models. If the differences are great enough, asteroseismic observations of pulsating PMS stars may be able to confirm the models. Indeed, F. Palla \& S. W. Stahler (2009, private communication) have indicated that they are currently investigating the oscillation properties of their models.

We are, in part, motivated to study the oscillations of PMS stars because we expect their interior structure to be relatively easily derived from the oscillation spectra, at least as predicted by classical PMS evolutionary models. In contrast, due to nuclear burning and core convection, the interiors of post-mainsequence stars have chemical gradients and discontinuities in the cores of the stars that result in significantly more complicated oscillation spectra.

In the structurally simplest of stellar models, (e.g., polytropic, classical PMS, and young post-main-sequence models) with $n \gg l$, the frequencies of the oscillation modes occur in a regular, nearly repeating pattern. For spherical models that assume small amplitude oscillations, the frequencies of the $p$-mode oscillations are given approximately by the asymptotic relation (Tassoul 1980), commonly written as

$$
v_{\mathrm{nl}} \cong\left(n+\frac{l}{2}+\frac{1}{4}+\beta\right) \Delta v-\left(A L^{2}-\varepsilon\right) \frac{\Delta v^{2}}{v_{\mathrm{nl}}},
$$

where

$$
\Delta v=\left[2 \int_{0}^{R} \frac{d r}{c}\right]^{-1}
$$

and

$$
A=\frac{1}{4 \pi^{2} \Delta v}\left[\kappa-\int_{0}^{R} \frac{d c}{d r} \frac{d r}{r}\right] .
$$

The constants $\beta, \varepsilon$, and $\kappa$ depend on the structure of the surface layers, $n$ is the radial order of the mode, $l$ is the angular degree of the mode, $c$ is the speed of sound, $R$ is the radius of the star, and $L=l+1 / 2$. It is easy to isolate the first- and second-order terms in the asymptotic expression by forming specific differences of the frequencies. The large frequency spacing, defined as,

$$
\Delta(n) \equiv v(n, l)-v(n-1, l),
$$

is (assuming $n \gg l$ ) approximately equal to the characteristic frequency spacing, $\Delta v$, which depends on the radius and sound speed in the surface layers. The small frequency spacing, defined as,

$$
\delta(n) \equiv v(n, l)-v(n-1, l+2) \cong \Delta v \int_{0}^{R} \frac{d c}{d r} \frac{d r}{r},
$$

isolates the second-order term, which depends primarily on the derivative of the sound speed in the interior.

It is common to plot an individual mode's oscillation frequency versus the frequency modulo the characteristic frequency. Called an echelle diagram, the modes of common angular order, $l$, line up, appearing as vertical sequences of points. Because no spatial information is usually available to determine the angular degree of the mode (except through line profile variability of rapidly rotating stars), the echelle diagram is used to identify the angular degree of the individual frequencies and establish the existence of nonradial oscillations. If the oscillation frequencies do not lie in well-defined linear sequences, associated with common angular degree, then either the observations are spurious, e.g., the artifacts of instrumental and observational periodicities and aliases, or the interior structure is significantly more complicated than assumed, possibly due to rapid rotation, strong magnetic fields, deviations from sphericity, and/or chemical discontinuities.

At the time of Zwintz (2008) only 36 pulsating PMS stars were known. This number has increased by a few owing to the observations from the Microvariability and Oscillations of Stars (MOST) space telescope (Walker et al. 2003) and is expected to increase again when results from the CoRoT space telescope (Baglin et al. 2002) are published. Usually the stars are associated, by proximity and velocity, with a young cluster. Both the age of the cluster and the star's position in the HR diagram are used to ascertain that the star is in its PMS phase of evolution. A post-main-sequence star similarly located in the HR diagram would have an age several orders of magnitude greater than the age of the cluster. Nearly all known PMS pulsators are found inside the $\delta$ Scuti instability strip, suggesting that the pulsation driving mechanism is similar to that of $\delta$ Scuti stars (Zwintz 2008).

Our first attempt to model pulsating PMS stars in a young cluster, NGC 6530 (Guenther et al. 2005, based on observations from the ground by Zwintz \& Weiss 2006), confirmed the nonradial character of the oscillations and, for this cluster, provided an independent (i.e., asteroseismic) determination of the reddening of each star. The results of observations by MOST of oscillating PMS stars in other young clusters will be reported separately.

In this paper, we consider six stars, here identified as V1, V2, V3, V4, V588 Mon, and V589 Mon, of which five are believed, owing to their proximity and space motion, to be members of the young open cluster NGC 2264. The seismic observations were obtained from the MOST satellite (Zwintz et al. 2009) and from a network of ground-based telescopes (Kallinger et al. 2008). Thorough discussions of the observations and data reductions for V1, V2, V3, and V4 can be found in Zwintz et al. (2009) and for the ground-based photometry of V588 Mon and V589 Mon in Kallinger et al. (2008).

In the following section, we provide an overview of our model-fitting strategy. In Section 3, we describe each star in turn, first discussing the three lowest luminosity stars, V2, $\mathrm{V} 3$, and V4, for which modeling was straightforward, and then the remaining stars, V588 Mon, V589 Mon, and V1, for which modeling was problematic. In Section 4, we present our interpretation of the results, and in Section 5, we conclude with some additional comments about the results.

\section{MODELS AND OSCILLATION FREQUENCY FITTING}

We use a relatively robust method to fit models to the observed oscillation spectra. The method was originally developed (Guenther \& Brown 2004) to deal with the sparse oscillation spectra obtained for stars and has been successfully applied to the interpretation of several asteroseismic observations: $\alpha$ Cen A (Guenther \& Brown 2004), $\eta$ Boo (Guenther et al. 2005; Guenther et al. 2007), Procyon (Guenther et al. 2007), and PMS stars in NGC 6383 (Zwintz et al. 2007). A large grid of stellar models, closely spaced in mass and age is constructed for a specific set of model parameters, such as composition and mixing-length parameter, using our stellar evolution code (YREC, Demarque et al. 2008). The adiabatic and nonadiabatic acoustic oscillation spectra of each model are computed 
(Guenther 1994). When presented with an observed oscillation spectrum, the grids of oscillation spectra are searched, systematically, for the model oscillation spectra that most closely match the observed frequencies.

The effectiveness of this strategy depends on the density of the grid in mass and age. If the models are too sparsely spaced in age then it is easy to miss abrupt changes to the oscillation frequencies caused by mode bumping during postmain-sequence evolution. Through extensive trials we have determined that the models must be spaced closely enough that the frequency differences for a given mode between adjacent models, in mass and age, are no more than a factor of 10 times the typical uncertainty in the observed frequencies, which for a two week observation run is of the order of $1 \mu \mathrm{Hz}$. For more evolved models with complicated interior structures, the denser the grid, the less likely the search for the best matching spectra will skip over the best-fitted region.

When the observed oscillation spectra only contain a couple of frequencies, as is the case for most of the previous generation of asteroseismic observations, more than one region of models can fit the observed frequencies. For example, if only two modes are observed there is the ambiguity of whether or not the modes are adjacent in radial order or angular order, or even if they are adjacent modes at all. Multiple solutions are inevitable. In these cases, other observational or theoretical constraints must be imposed.

We use the following equation for $\chi^{2}$ to quantify how well the observed spectrum matches each model spectrum in the grid:

$$
\chi^{2}=\frac{1}{N} \sum_{i=1}^{N} \frac{\left(v_{\mathrm{obs}, i}-v_{\mathrm{mod}, i}\right)^{2}}{\sigma_{\mathrm{obs}, i}^{2}+\sigma_{\mathrm{mod}, i}^{2}},
$$

where $v_{\mathrm{obs}, i}$ is the observed frequency for the $i$ th mode, $v_{\mathrm{mod}, i}$ is the corresponding model frequency, $\sigma_{\mathrm{obs}, i}$ is the observational uncertainty for the $i$ th mode, and $N$ is the total number of modes that match the observed frequencies. The model uncertainty $\sigma_{\text {mod }, i}$ can be estimated from fits of models to the solar oscillation spectrum (Guenther \& Brown 2004). The discrepancy between our solar model frequencies and the observed frequencies is approximately $0.008 \%$ for $n<20$ rising to $0.3 \%$ for $n>30$. For the frequencies of the PMS stars discussed in this paper, the model uncertainty is more than an order of magnitude smaller than the observational uncertainties of $\sim 0.3 \mu \mathrm{Hz}$. Therefore, in the present case, we have chosen to ignore this term. We seek models that minimize $\chi^{2}$.

Our pre- and post-main-sequence models were constructed using the Yale Stellar Evolution Code (YREC; Demarque et al. 2008) with masses ranging from $0.81 M_{\odot}$ to $4.99 M_{\odot}$ in steps of $0.01 M_{\odot}$. Each evolutionary track comprises approximately 1000 models, totaling $\sim 400,000$ models in each grid.

The model grids were constructed using the OPAL98 (Iglesias \& Rogers 1996) and Alexander \& Ferguson (1994) opacity tables, and the Lawrence Livermore equation of state tables (Rogers 1986; Rogers et al. 1996). The mixing length parameter sets the temperature gradient in convective regions according to the Böhm-Vitense mixing-length theory (Böhm-Vitense 1958). All tracks were computed with the mixing length parameter obtained from a solar calibrated model constructed using the same physical parameters as the PMS grid (for YREC, this corresponds to $\alpha=1.8$ ). The atmosphere is gray and in the Eddington approximation. Nuclear reaction cross sections are from Bahcall et al. (2001). To avoid complicating the interpretation of the model physics, we do not include the effects of mass loss or infall, convective overshoot, rotation, nor the gravitational settling of helium and heavy elements. Some of these omitted physics, as we will argue, may be very important for the full and correct interpretation of the observed oscillation spectra but our first step is to analyze and understand the strengths and limitations of our classical PMS models.

Our PMS models begin as completely convective LaneEmden spheres (Lane 1869; Chandrasekhar 1957) and are evolved from the Hayashi track to the ZAMS. They are not models built up by mass infall as in Palla \& Staller. Our models compare well with the evolutionary tracks of D'Antona \& Mazzetelli (1994), which also do not account for mass buildup through infall. Although the grid itself includes all models on the Hayashi track, in our analysis we will assume only those models evolved below the birthline, as computed by Palla \& Stahler, are viable models. The post-main-sequence evolutionary tracks from which the post-main-sequence grid was constructed are similar to standard reference tracks of the Yonsei-Yale group (Yi et al. 2003) except that we have computed an order of magnitude more models for each evolutionary track.

Adiabatic and nonadiabatic $p$-modes for $0 \leqslant l \leqslant 3$ and 0 $\leqslant n \leqslant 35$ were calculated for all models in the grid. The pulsation spectra were computed using the stellar pulsation code of Guenther (1994), which solves the linearized, nonradial, nonadiabatic pulsation equations using the Henyey relaxation method. The nonadiabatic solutions include radiative energy gains and losses but do not include the effects of convection. Here we only utilize the adiabatic frequencies from the grid.

\section{ASTEROSEISMIC ANALYSIS}

The asteroseismic data used in our analysis originate from both MOST and multi-site ground-based observations. For stars V1, V2, V3, and V4 in open cluster NGC 2264, we use the MOST results presented in Zwintz et al. (2009) and for V588 Mon and V589 Mon, we use both the MOST results from T. Kallinger et al. (2009, private communication) that are currently being prepared for publication and his multi-site ground-based results (Kallinger et al. 2008). The cited papers also contain a summary of current available photometric data on each star, which we will incorporate in our model analysis. All of the stars are located away from the center of the cluster. The MOST data are available for download from the MOST Public Data Archive, (http://www.astro.ubc.ca/MOST/data/data.html) or upon request to the authors. The MOST 2006 observations were made from 2006 December 7 to 2007 January 3 and provide $\sim 23 \mathrm{~d}$ of coverage. The ground-based observations of V588 Mon and V589 Mon were made from 2002 November to 2003 February and provide $\sim 9 \mathrm{~d}$ of coverage.

In Figure 1, we show a theoretical HR diagram with the locations of the six stars along with their estimated uncertainties as determined from their magnitudes and photometric color indices under the assumption that they are members of the cluster NGC 2264. Here we have assumed that NGC 2264 is $760 \pm 80 \mathrm{pc}$ away and the stars within have negligible reddening (Zwintz et al. 2009). A sample of our standard PMS evolutionary tracks (solar composition) is drawn (solid line) and labeled by their masses. Several post-main-sequence tracks are also shown (dashed line). The birthline from Palla \& Stahler (1993), corresponding to a mass accretion rate of $10^{-5} M_{\odot} \mathrm{yr}^{-1}$, is drawn in bold. PMS stars located above this line are believed to be hidden from view by the primordial dust and gas cloud from which the stars are formed. 


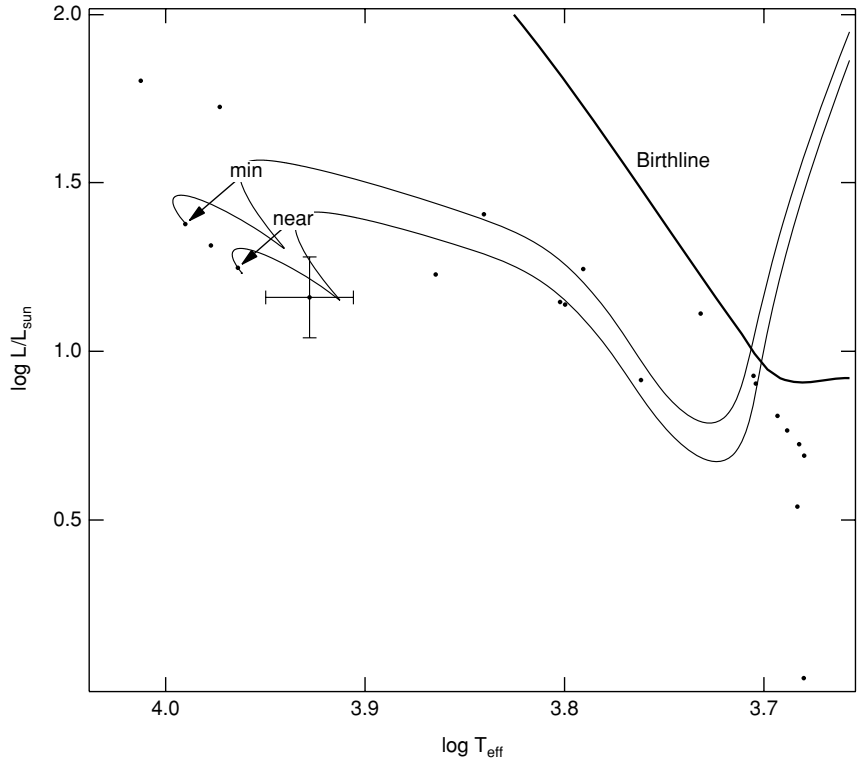

Figure 1. Theoretical HR diagram showing the locations of the six PMS stars associated with NGC 2264. PMS (solid lines) and post-main-sequence tracks (dashed lines) are shown for selected masses (as labeled).

We note that the proper motion of V1 (Hog et al. 1998) does not match the proper motion of NGC 2264 (Kharchenko 2001) hence the probability that it is a member of the cluster is low compared to the other stars (Zwintz et al. 2009). Therefore, in fact, its distance and luminosity must be considered unknown. Regardless, we will carry out our analysis assuming that it is a member to see if the seismic data can help refute its membership.

The stars divide into two groups, V2, V3, and V4 clustered near the $2 M_{\odot}$ PMS track and V1, V588 Mon, and V589 Mon clustered near the $3 M_{\odot}$ track. Immediately we deduce a spread in age among these stars. To have arrived at their current location in the HR diagram at the current epoch, the higher mass stars located early on in their evolution will have to have formed more recently than the lower mass stars located closer to the ZAMS. This does not imply that low-mass star formation has stopped in the cluster and that only high-mass star formation is continuing (see Stahler 1983, for a thorough discussion of this point). Finally, we note that because the rate of evolution of a star is directly proportional to its luminosity, the more massive stars have evolved more quickly, hence, have only recently emerged from the accretion phase above the birthline.

The number of independent frequencies identified in the oscillation spectra of the stars obtained from MOST varies from only two for V2 to a dozen for V589 Mon.

In the following subsections, we present model analysis of the asteroseismic data for each star beginning with V2, the star with the least complicated oscillation spectrum, continuing through to V588 Mon and V589 Mon, the two stars with the most complicated oscillation spectra, and ending up with V1, our most enigmatic star.

\subsection{V2 (HD261711)}

V2 (HD 261711) is a relatively faint star $(V=11.32)$ in which the MOST data reveal two significant frequencies (see Table 1) after stray light artifacts modulated by the MOST satellite orbit have been removed. Note that the removal of satellite orbital harmonics and other stray light modulation terms may also remove intrinsic modes, but this is preferable to including any frequency of nonstellar origin in the analysis, as such a situation
Table 1

Selected Observed Frequencies

\begin{tabular}{|c|c|c|c|}
\hline Star & Label & Frequency $(\mu \mathrm{Hz})$ & Amplitude (mmag) \\
\hline \multirow[t]{2}{*}{$\mathrm{V} 2$} & $\mathrm{~F} 1$ & $711.52 \pm 0.05$ & 5.0 \\
\hline & $\mathrm{F} 2$ & $671.41 \pm 0.06$ & 1.0 \\
\hline \multirow[t]{4}{*}{ V3 } & $\mathrm{F} 1$ & $401.55 \pm 0.05$ & 3.7 \\
\hline & $\mathrm{F} 2$ & $249.8 \pm 0.2$ & 1.0 \\
\hline & F3 & $397.7 \pm 0.2$ & 0.9 \\
\hline & $\mathrm{F} 4$ & $790.7 \pm 0.2$ & 0.8 \\
\hline \multirow[t]{3}{*}{$\mathrm{V} 4$} & $\mathrm{~F} 1$ & $407.45 \pm 0.05$ & 9.4 \\
\hline & $\mathrm{F} 2$ & $380.8 \pm 0.1$ & 4.6 \\
\hline & F3 & $377.3 \pm 0.1$ & 4.5 \\
\hline \multirow[t]{8}{*}{ V588 Mon } & $\mathrm{F} 1$ & $59.5 \pm 0.3$ & 8.7 \\
\hline & $\mathrm{F} 2$ & $126.6 \pm 0.3$ & 5.9 \\
\hline & F3 & $60.9 \pm 0.3$ & 5.3 \\
\hline & $\mathrm{F} 4$ & $81.4 \pm 0.3$ & 3.3 \\
\hline & F5 & $64.2 \pm 0.3$ & 2.2 \\
\hline & F6 & $96.0 \pm 0.3$ & 2.0 \\
\hline & F7 & $88.7 \pm 0.3$ & 1.9 \\
\hline & F8 & $127.1 \pm 0.3$ & 0.6 \\
\hline \multirow[t]{12}{*}{ V589 Mon } & $\mathrm{F} 1$ & $75.1 \pm 0.3$ & 14.0 \\
\hline & $\mathrm{F} 2$ & $80.9 \pm 0.3$ & 8.7 \\
\hline & $\mathrm{F} 3$ & $96.1 \pm 0.3$ & 3.3 \\
\hline & $\mathrm{F} 4$ & $66.7 \pm 0.3$ & 3.3 \\
\hline & F5 & $100.7 \pm 0.3$ & 2.4 \\
\hline & F6 & $118.0 \pm 0.3$ & 2.3 \\
\hline & F7 & $109.6 \pm 0.3$ & 1.5 \\
\hline & F8 & $72.7 \pm 0.3$ & 1.6 \\
\hline & F9 & $107.8 \pm 0.3$ & 1.5 \\
\hline & F10 & $118.8 \pm 0.3$ & 1.5 \\
\hline & F11 & $127.2 \pm 0.3$ & 1.4 \\
\hline & $\mathrm{F} 12$ & $77.5 \pm 0.3$ & 0.8 \\
\hline \multirow[t]{7}{*}{ V1 } & $\mathrm{F} 1$ & $226.8 \pm 0.1$ & 2.8 \\
\hline & $\mathrm{F} 2$ & $126.5 \pm 0.1$ & 2.8 \\
\hline & F3 & $139.2 \pm 0.1$ & 2.7 \\
\hline & $\mathrm{F} 4$ & $113.3 \pm 0.2$ & 1.8 \\
\hline & F5 & $243.3 \pm 0.2$ & 1.8 \\
\hline & F6 & $194.3 \pm 0.2$ & 1.7 \\
\hline & F7 & $156.9 \pm 0.2$ & 1.7 \\
\hline
\end{tabular}

could lead to wrong results. From Zwintz et al. (2009), we take $T_{\text {eff }}=8500 \pm 300 \mathrm{~K}$ and $\log L / L_{\odot}=1.16 \pm 0.12$.

It is not usually possible to fit a unique model with only two modes. Many model solutions are possible. Figure 2 shows in an HR diagram the location of all the models in the grid whose oscillation spectra can be matched to the two observed frequencies within the uncertainties, or more specifically, with $\chi^{2} \leqslant 1$ (as defined in Section 2). For these fits, the model oscillation spectra include $l=0,1,2$, and $3 p$-modes (whose radial orders, $n$, include all modes from the fundamental at $n=$ 0 to the acoustic cutoff frequency). We were not able to find any model fits using only radial $(l=0)$ modes. The model with the lowest $\chi^{2}$, labeled min in Figure 2, has $\chi^{2}=0.08$. The model labeled near corresponding to the model (with $\chi^{2} \leqslant 1$ ) closest to the observed position of the star in the HR diagram has $\chi^{2}=$ 0.97. PMS evolutionary tracks are shown in Figure 2 for these two model fits.

In Figure 3, we present an echelle diagram of the observed (large open circles) and model frequencies of the min and near models. Owing to the nearly constant frequency separating $p$-modes of similar $l$ adjacent in $n$-, $p$-modes of common $l$ appear along vertical curves in the echelle diagram.

Because $p$-modes are regularly spaced in frequency with a spacing that depends on the radius of the star, when matching 


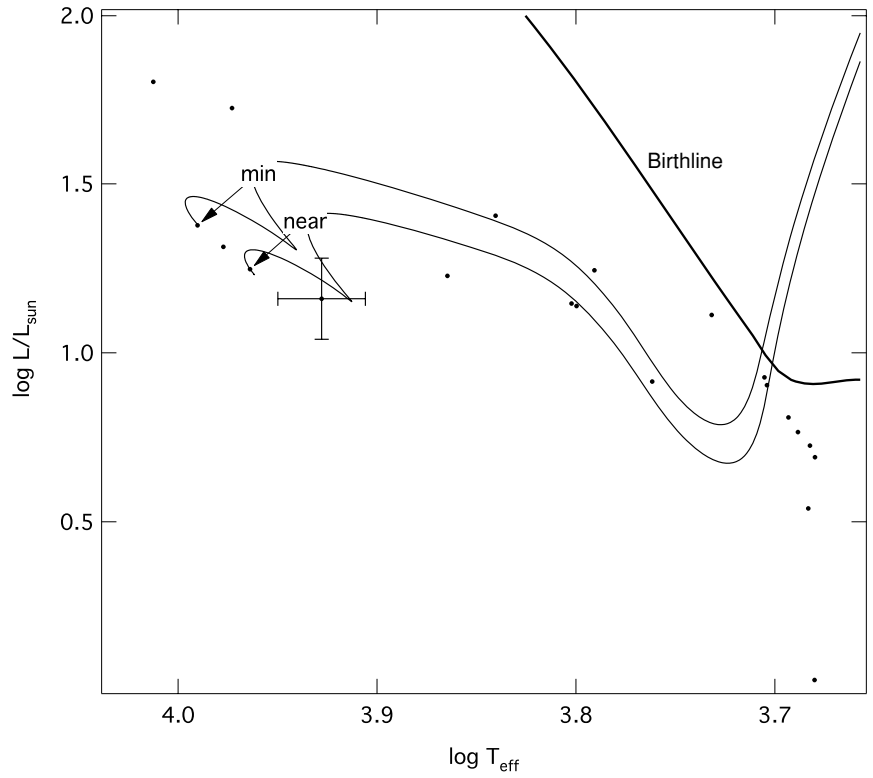

Figure 2. Theoretical HR diagram showing all models from a grid of $\sim 400,000$ PMS models whose $p$-mode oscillation spectra match the observed oscillation frequencies of V2 with $\chi^{2} \leqslant 1$.

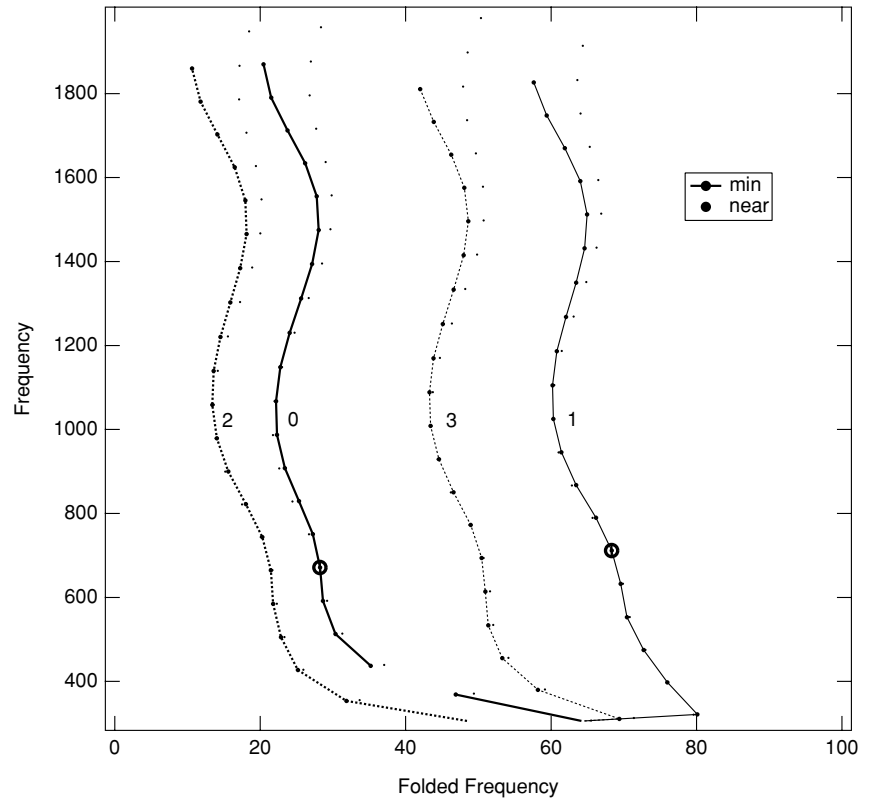

Figure 3. Echelle diagram (frequency vs. frequency modulo $80.4 \mu \mathrm{Hz}$ ) showing the two observed frequencies (bold open circles) of V2 along with the $p$-mode oscillation frequencies for the min model (connected points, model with the best fitting $p$-mode frequencies) and the near model (model with $\chi^{2} \leqslant 1$ and closest to the location of $\mathrm{V} 2$ in the HRD diagram). Labels denote the angular degree of the model modes.

model spectra to observations, the models tend to fall along constant radius sequences in the HR diagram. This behavior will be noticeable when we have a greater number of possible combinations of modes to define the spacings between the modes.

Table 2 summarizes the physical characteristics of the $\mathrm{min}$ and near model fits for star V2. "Conv. Core" is the mass of the star contained in the central convection zone, and "Conv. Env." is the radius fraction of the base of the convective envelope. The age is the time to evolve from the top of the Hayashi track to the model's current position. The birthline age is the difference

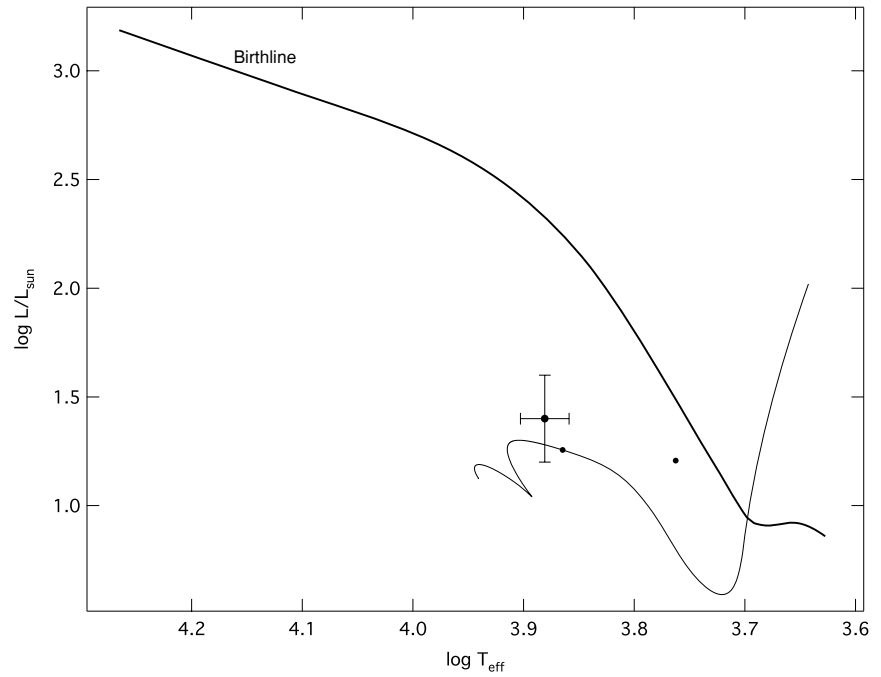

Figure 4. Theoretical HR diagram showing all models (the two points) from a grid of $\sim 400,000$ PMS models whose $p$-mode oscillation spectra match the observed oscillation frequencies of V3 with $\chi^{2} \leqslant 1$. An evolutionary track is drawn through the model (of the two) with the lowest $\chi^{2}$.

between the model's current age and its age as it crosses the birthline. Since the star evolves very rapidly down the Hayashi track, for lower mass stars $\left(<3 M_{\odot}\right)$ the uncertainties associated with where and when the star begins its evolution are small compared to the star's total evolutionary time and in the case of the two models for $\mathrm{V} 2$, the difference is only $\sim 5 \%$ the age of the star.

Age estimates for NGC 2264 itself range from $3 \mathrm{Myr}$ (Walker 1956) to $10 \mathrm{Myr}$ (Sagar et al. 1986); hence, the ages of our two representative model fits to V2's seismic data are consistent with the age of the cluster. The age of the cluster is measured from the time the most massive stars arrive on the ZAMS. The massive stars in NGC 2264 have already evolved off the main sequence. Indeed, it is the luminosity of this turnoff point, calibrated by grids of stellar model isochrones that is used to determine the cluster age. The lower mass star V2, on the other hand, has not yet completed its PMS evolution. It is only $\sim 1$ Myr away from arriving on the ZAMS and beginning nuclear burning.

V2's convective envelope, which on the Hayashi track extended all the way from the surface to the core, has shrunk to a thin layer near the surface.

\subsection{V3 (HD261387)}

Four significant intrinsic frequencies were identified in the MOST data of this star (Table 1). Here we adopt values cited by Zwintz et al. (2009) with $V=10.60 \mathrm{mag}, T_{\text {eff }}=7600 \pm$ $350 \mathrm{~K}$, and $\log L / L_{\odot}=1.4 \pm 0.2$. And we note that $v \sin i=$ $140 \mathrm{~km} \mathrm{~s}^{-1}$.

From our grid of $\sim 400,000$ PMS models, only two models were found with oscillation spectra that match the four observed modes with $\chi^{2} \leqslant 1$ (see Figure 4). Figure 4 shows the location of the two models (points without error bars) with respect to the observed location of V3 (point with error bars). A PMS track passing through the best-fitting model $\left(\chi^{2}=0.5\right)$ is also shown. The best-fitting model lies within the uncertainties of the V3's position in the theoretical HR diagram.

Table 2 contains a summary of the properties of the best-fitting model. The model's mass and age are similar, as expected based on its location in the HR diagram, to the models for V2. 
Table 2

Model Fit Characteristics

\begin{tabular}{lcccccccc}
\hline \hline \multicolumn{1}{c}{ Model } & Mass $^{\mathrm{a}}$ & Age $^{\mathrm{b}}$ & Birthline Age $^{\mathrm{b}}$ & $\log L / L_{\odot}$ & $\log R / R_{\odot}$ & $\log _{\text {eff }}$ & Conv. Core $^{\mathrm{a}}$ & Conv. Env. $^{\mathrm{c}}$ \\
\hline V2 min & 2.22 & 14.4 & 13.8 & 1.377 & 0.2320 & 3.9902 & 0.335 & 0.994 \\
V2 near & 2.04 & 9.0 & 8.5 & 1.247 & 0.2199 & 3.9638 & 0.217 & 0.995 \\
V3 best & 1.92 & 6.7 & 6.2 & 1.256 & 0.4225 & 3.8646 & 0.0 & 0.918 \\
V4 near & 1.88 & 7.5 & 7.0 & 1.261 & 0.3553 & 3.8995 & 0.147 & 0.992 \\
V588 Mon near & 3.39 & 1.43 & 0.17 & 1.909 & 0.7738 & 3.8522 & 0.0 & 0.989 \\
V589 Mon near & 3.04 & 1.91 & 1.08 & 1.755 & 0.7212 & 3.8402 & 0.0 & 0.990 \\
V1 near no rot & 3.77 & 1.1 & & 2.069 & 3.868 & & & \\
V1 min no rot & 4.42 & 0.7 & & 2.426 & 3.946 & & & \\
V1 near rot & 3.53 & 1.4 & & 2.046 & 1.762 & & & \\
V1 min rot & 3.30 & 1.4 & & 1.762 & 3.806 & & & \\
\hline
\end{tabular}

Notes.

${ }^{a}$ In units of $M_{\odot}$.

b In units of Myr.

${ }^{\mathrm{c}}$ In units of $R / R_{\odot}$.

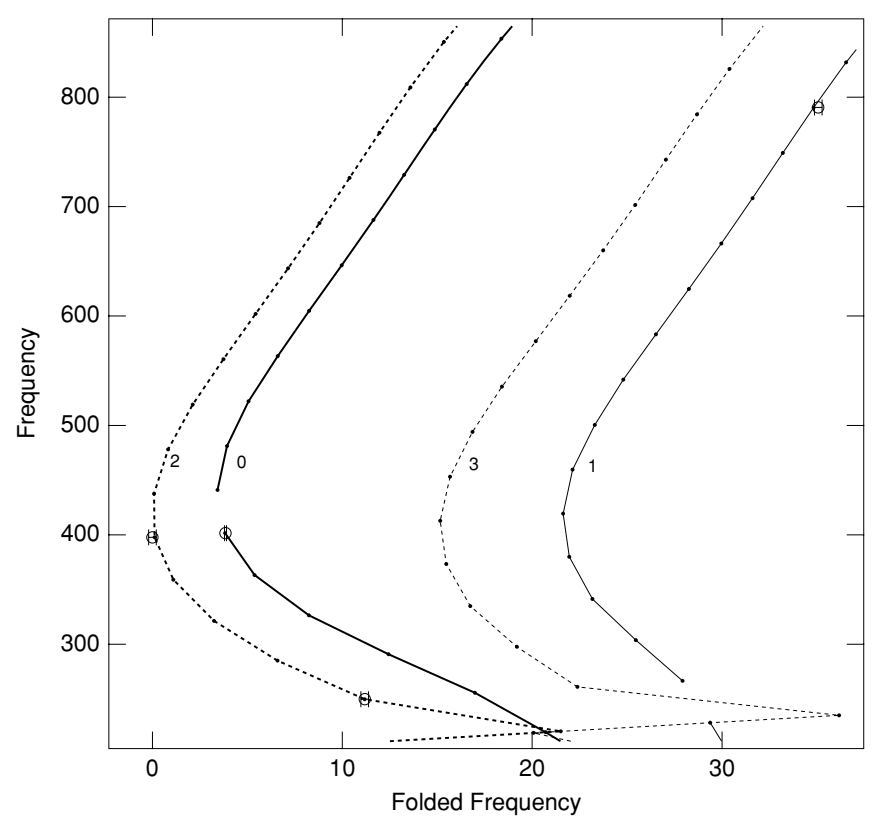

Figure 5. Echelle diagram (frequency vs. frequency modulo $39.8 \mu \mathrm{Hz}$ ) showing the four observed frequencies (open circles with uncertainty bars) of V3 along with the $p$-mode oscillation frequencies for best-fitting model. Labels denote the angular degree of the model modes.

In Figure 5, we show the echelle diagram for the observed (large open circles) and best-fit model frequencies (points connected by lines). The frequency uncertainties are also indicated for the observed data points.

In summary, it appears that both the quality of the oscillation fit and the agreement with other observables support our model fit to V3 and, thus, confirm the age of the cluster and its distance. Unfortunately, we must note that the star has a very high $v \sin i$, which must affect the frequencies. Although we see no evidence of splitting in the limited number of observed frequencies, the possibility remains that we are fitting a retrograde or prograde frequency or that higher order, rotation dependent, corrections to the frequencies are important. Finally, we note that the frequencies can only be fit when nonradial modes are included.

\subsection{V4}

MOST observations revealed the three frequencies listed in Table 1. Based on Zwintz et al. (2009), we adopt $T_{\text {eff }}=7600 \pm$ $500 \mathrm{~K}$ and $\log L / L_{\odot}=1.12 \pm 0.1$.
We were not able to find any model oscillation spectra with $l=0,1,2$, and $3 p$-modes that fit all three observed frequencies within the uncertainties. The two best-fitting models with $\chi^{2}=\sim 5$ lie far from the observed position of V4 in the theoretical HR diagram. We attribute the difficulty in finding a fit to the closeness of the two frequencies F2 and F3. Whenever two or more frequencies are very close together in frequency, there are several possible interpretations. If the mode varies in amplitude over the duration of the observations then, because Fourier analysis assumes the oscillation amplitudes are constant, the amplitude spectrum will yield multiple peaks centered on the frequency of the varying amplitude mode. There is also the possibility that the mode is part of a multiplet, with splitting caused by rotation, spherical distortion, or magnetic fields. Regardless of the cause, when we cannot find a model fit to all frequencies in an apparent multiplet, we proceed by excluding all but one of the frequencies, assuming that the excluded frequencies are outside the domain of our modeling parameters. For V3, we present the results of fitting frequencies F1 and F2. Similar results are obtained when fitting $\mathrm{F} 1$ and $\mathrm{F} 3$.

By fitting just the two frequencies $\mathrm{F} 1$ and $\mathrm{F} 2$ we found a model, identified as near, that has both a low $\chi^{2}$ and is close to V4's position in the HR diagram. The two modes are fit by $l=0$ and $1 p$-modes. The F2 and F3 frequencies lie close to the $l=1 p$-mode sequence, which is consistent with the possibility that F2 and F3 are part of a rotational triplet, since radial modes are not split by rotation. In Table 2, we summarize the model parameters of the near model. The age of the model is consistent with the age of NGC 2264 and the asteroseismic ages derived for V2 and V3.

If the separation between F2 and F3 $(3.4 \mu \mathrm{Hz})$ is an $l=1$ rotational splitting, then the equatorial rotational velocity for the near model (solid body rotation) is $\sim 34 \mathrm{~km} \mathrm{~s}^{-1}$.

In summary, for V4 we are only able to fit two of the three observed frequencies and, at the same time, produce models that lie close to V4's position in the HR diagram. The close spacing between two of the frequencies (F2 and F3) could be caused by rotation. Less likely is our model fit to all three frequencies. This fit is poorer seismically, with $\chi^{2}=\sim 5$, and is far from V4's location in the HR diagram.

\subsection{V588 Mon}

We used the satellite data from the MOST 2004 run, the MOST 2006 run (Kallinger 2009), and the data from the multi- 


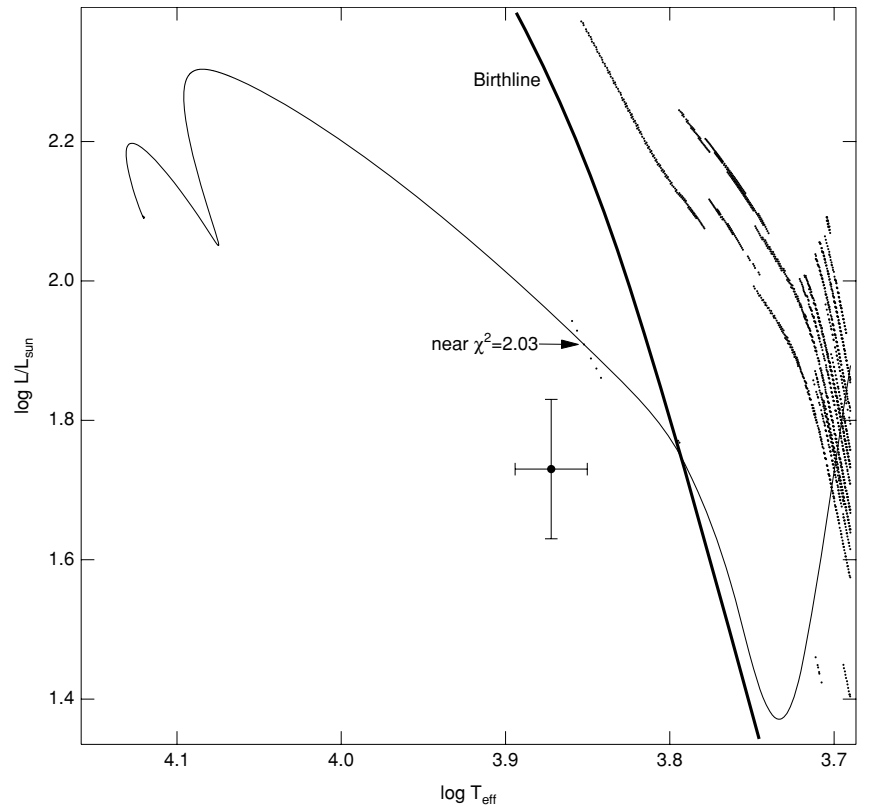

Figure 6. Theoretical HR diagram showing all models from our PMS grid whose $p$-mode oscillation spectra match the six selected oscillation frequencies of V588 Mon with $\chi^{2} \leqslant 2$. The PMS evolutionary track corresponding to the near model is shown.

site ground-based campaign (Kallinger et al. 2008). The MOST data were reduced as described in Zwintz et al. (2009).

We winnowed the three lists of frequencies down to those frequencies that are common to all three data sets because we want to be as certain as possible that all our frequencies are intrinsic to the star. Indeed, if even one frequency is nonintrinsic then the oscillation spectra search and model-fitting strategy either will fail outright or (worse) yield false model fits. Also, we note that if even one frequency is outside the domain of our modeling parameters (e.g., a rotational split side lobe) then the model fits will be skewed by an amount dependent on how far from the standard model predictions the observed frequency is. This could be significant since the measured $v \sin i=130 \pm$ $20 \mathrm{~km} \mathrm{~s}^{-1}$ (see Kallinger et al. 2008).

The MOST 2004 and MOST 2006 runs reveal many frequencies, but the majority of them are linear combinations of a smaller number. The two time series have 10 significant frequencies in common. Of these, eight are also found in the Kallinger et al. (2008) observations. In Table 1, we list the eight frequencies and their amplitudes from the MOST 2004 data. The list reveals several close pairs of modes, F1, F3 and F2, F8, with separations of $1.45 \mu \mathrm{Hz}$ and $0.54 \mu \mathrm{Hz}$, respectively.

From Kallinger et al. (2008), we adopt $T_{\text {eff }}=7450 \pm 350 \mathrm{~K}$, and $\log L / L_{\odot}=1.73 \pm 0.1$.

We were unable to find any oscillation spectra in the model grid that fits all eight observed frequencies. As a consequence, we investigated a smaller sample of the observed frequencies. We removed the $\mathrm{F} 3$ and $\mathrm{F} 8$ frequencies arguing that because they are close to the F1 and F2 frequencies, respectively, they could represent rotationally split modes or could be an artifact of the application of a Fourier analysis to an amplitude-varying mode. In both cases, our model-fitting program lacks the robustness to handle these situations.

We found only a few model fits to the F1, F2, F4, F5, F6, and F7 frequencies that lie below the birthline. In Figure 6, we plot all our models that match the observed six frequencies with $\chi^{2} \leqslant 2.1$. At this slightly higher cutoff in $\chi^{2}$, we pick up a

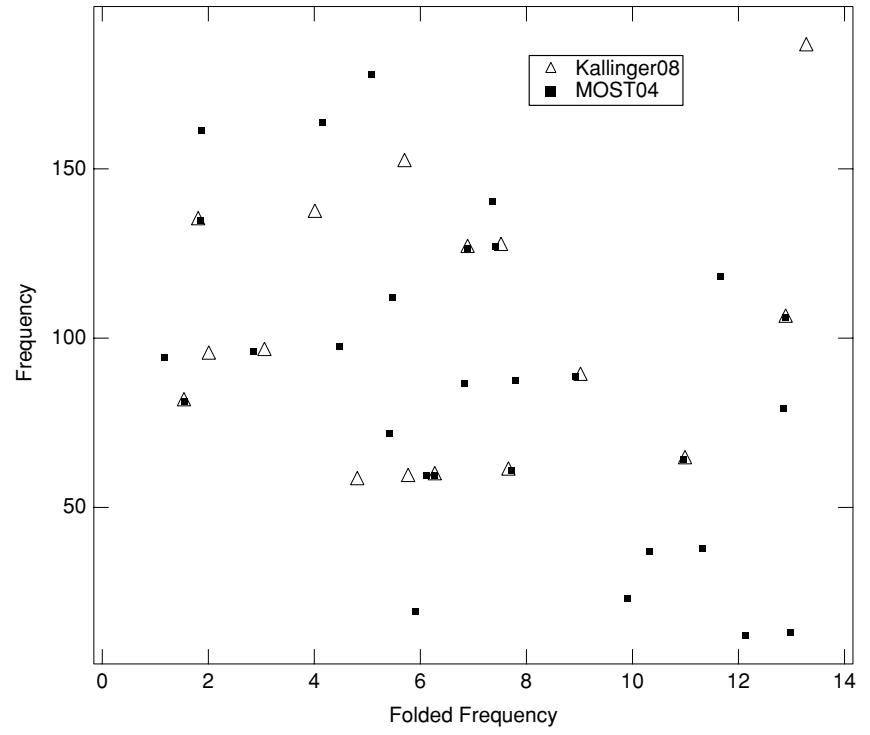

Figure 7. Echelle diagram (frequency vs. frequency modulo $13.3 \mu \mathrm{Hz}$ ) showing all of the observed frequencies of V588 Mon from the satellite observations (MOST04) and the multi-site ground-based observations (Kallinger et al. 2008).

couple of models below the birthline, near the observed position of V588 Mon in the theoretical HR diagram. The closest model to V588 Mon in the HR diagram is labeled near. Its spectrum fits the observed spectrum with $\chi^{2}=2.03$.

Because the six fitted frequencies appear in both MOST 2004 and 2006 data and the ground-based observations of Kallinger et al. (2008), the frequencies are most likely to be intrinsic to the star, so the poor model fit is not a result of spurious nonstellar frequencies. We list the properties of the best-fitting model in Table 2.

The age implied by the model is much younger than the ages derived for stars V2, V3, and V4 (and for NGC 2264). In addition, it is more luminous than predicted under the assumption that it is a member of NGC 2264. We are unable to confirm V588 Mon's membership in the cluster using the star's seismic data.

If the separation between F1 and F3 $(1.45 \mu \mathrm{Hz})$ is an $l=1$ rotational splitting, then the corresponding equatorial rotational velocity for the near model (solid body rotation) is $\sim 38 \mathrm{~km} \mathrm{~s}^{-1}$ and if the separation between F2 and F8 $(0.54 \mu \mathrm{Hz})$ is an $l=1$ rotational splitting, then the corresponding equatorial rotational velocity for the near model (again, solid body rotation) is 14 $\mathrm{km} \mathrm{s}^{-1}$. These results cannot be reconciled with the observed $v \sin i=130 \mathrm{~km} \mathrm{~s}^{-1}$. We note that the small frequency spacing, $\delta(l) \equiv v(n, l)-v(n-1, l+2)$ for V588 Mon is $1.7 \pm 0.3 \mu \mathrm{Hz}$, i.e., the small frequency spacing is very close to the frequency separating F1 and F3.

In Figure 7, we show all of the significant frequencies identified in the MOST 2004 data and the Kallinger et al. (2008) data. Clearly, there is no obvious alignment of the modes in common $l$-valued sequences.

In summary, we are unable to fit the observed frequencies of V588 Mon even to a set of frequencies limited to only those common to all three data sets and which excludes close pairs. Additionally, the rotation velocities implied by the close pairs of frequencies is too low to be consistent with the observed $v \sin i=130 \mathrm{~km} \mathrm{~s}^{-1}$. Indeed, based on the large $v \sin i$, it is more likely that the actual rotational splitting is comparable to the large spacing for stars in this part of the HR diagram 


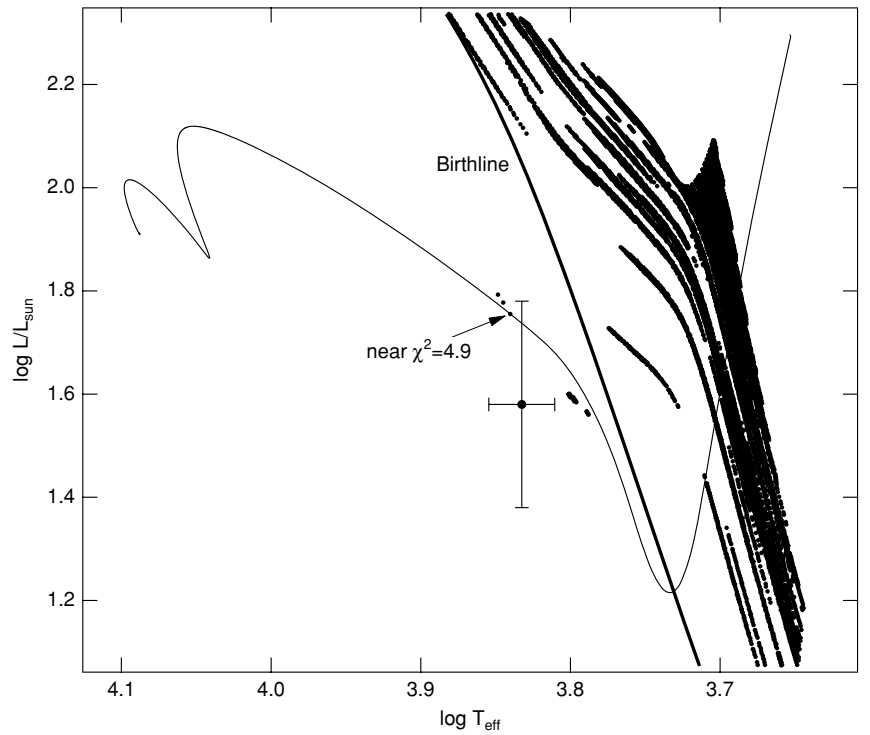

Figure 8. Theoretical HR diagram showing all models from our PMS grid whose p-mode oscillation spectra match the eight selected oscillation frequencies of V589 Mon with $\chi^{2} \leqslant 5$. The PMS evolutionary track corresponding to the near model is shown.

( $\sim 20 \mu \mathrm{Hz}$ ) and this could significantly complicate the interpretation and identification of the observed frequencies. Furthermore, as will be the case for all rapidly rotating stars, the higher order corrections, a function of rotation and inclination, could be important.

\subsection{V589 Mon}

The modeling of V589 Mon is similar to V588 Mon. We have three data sets: the satellite observations from MOST 2004, MOST 2006 (T. Kallinger et al. 2009, private communication), and the ground based observations (Kallinger et al. 2008). For our model fitting, we selected only those frequencies in common to all three data sets, yielding the 12 frequencies listed in Table 1 .

From Kallinger et al. (2008), we adopt $T_{\text {eff }}=6800 \pm 350 \mathrm{~K}$, $\log L / L_{\odot}=1.58 \pm 0.10$, and $v \sin i=60 \pm 10 \mathrm{~km} \mathrm{~s}^{-1}$.

We were unable to find any model fits to all 12 observed frequencies. We identified the following closely spaced frequencies multiplets, (F1, F8, F12), (F7, F9), and (F6, F10) with separations $2.36 \mu \mathrm{Hz}, 1.81 \mu \mathrm{Hz}$, and $0.82 \mu \mathrm{Hz}$, respectively, and retained only one frequency from each multiplet along with the rest of the frequencies for model fitting; i.e., we tried to fit models to the eight frequencies F1, F2, F3, F4, F5, F6, F7, and F11.

We obtained several model fit solutions with $\chi^{2} \sim 5$ near V589 Mon's location in the theoretical HR diagram (see Figure 8). No lower $\chi^{2}$ fits were found below the birthline. The model nearest (in the HR diagram) to V589 Mon, with $\chi^{2}=$ 4.9 , has the oscillation frequencies shown in the echelle diagram, Figure 9. Figure 9 also shows all the MOST 2004 and Kallinger et al. (2008) frequencies. The eight selected frequencies are indicated by large open squares. The fit is poor. Although there is a sense that the observed modes do line up in vertical sequences, the model $p$-modes do not fit the observed modes within the uncertainties $( \pm 0.3 \mu \mathrm{Hz})$.

The mass of the best-fit model is $3.04 M_{\odot}$ (see Table 2), which means that the star has only recently dropped below the birthline. The birthline age depends sensitively on the location of

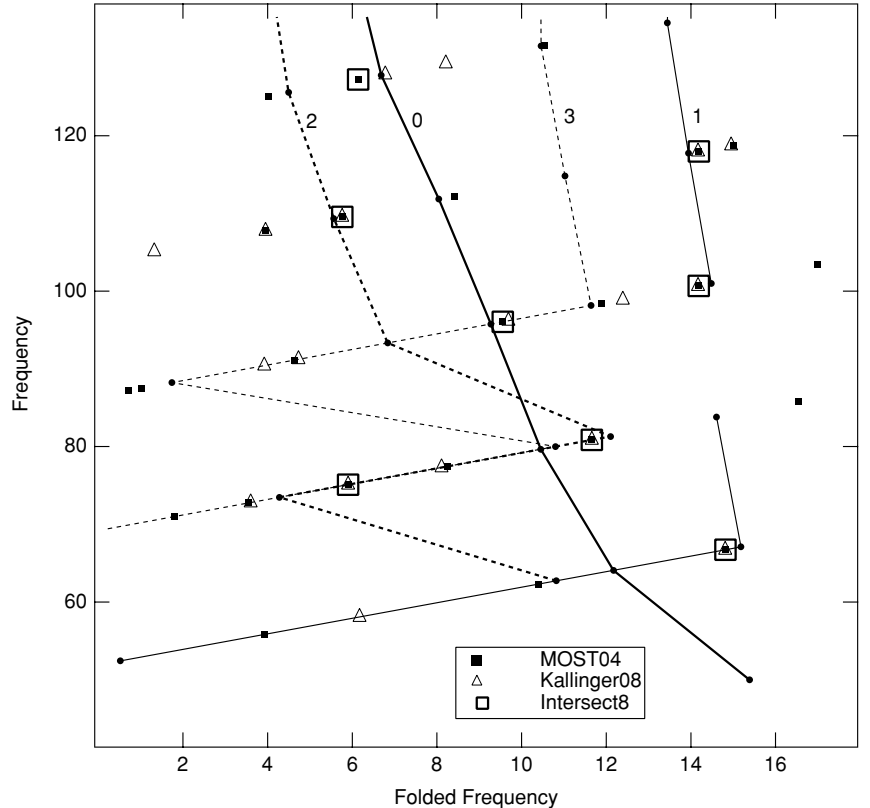

Figure 9. Echelle diagram (frequency vs. frequency modulo $17.3 \mu \mathrm{Hz}$ ) showing all of the observed frequencies of V589 Mon from the satellite observations (MOST04) and the multi-site ground-based observations (Kallinger et al. 2008). The eight frequencies selected for modeling are also indicated (Intersect8). The $p$-mode frequencies of the near model are plotted with labels denoting the angular degree of the model modes.

the birthline. Regardless, the star has only just recently emerged from its primordial nebula.

The multiplet frequencies, especially the triplet (F1, F8, F12), suggest rotational splitting. If all the multiplets are due to rotation, then the interior rotation would have to have a steep gradient in order to explain the different splittings. For the near model, the triplet's spacing, assuming the mode is an $l=1$, implies a solid body rotational velocity of $54 \mathrm{~km} \mathrm{~s}^{-1}$ at the equator. This is not inconsistent with the observed $v \sin i$. If the other multiplets are rotational splittings, then their rotational velocities are too low to be consistent with the observed $v \sin i$.

\subsection{V1 (HD261230)}

We finally arrive at V1, the star, which owing to its space velocity is unlikely to be a member of NGC 2264. Here we review the case of $\mathrm{V} 1$ in detail because it highlights many of the issues we face when trying to interpret the oscillation spectrum of a field star.

Zwintz et al. (2009) inform us that the star's proper motion in declination is more than 4 times that of NGC 2264, making it an unlikely member of the cluster. The spectral type, determined from DDO spectroscopy (Pribulla et al. 2009), is F2V. Zwintz et al. (2009) compared the DDO observations to synthetic spectra and derive $T_{\text {eff }}=7700 \pm 200 \mathrm{~K}$ and a rotational velocity $v \sin i=95 \pm 5 \mathrm{~km} \mathrm{~s}^{-1}$. With $V=9.39 \mathrm{mag}$ and assuming that it is the same distance from us as is NGC 2264, we derive $\log L / L_{\odot}=1.91 \pm 0.10$.

Of the 35 peaks found in the MOST 2006 data (Zwintz et al. 2009), seven survived orbit harmonic and alias filtering. The seven final frequencies are listed in Table 1 . We see immediately a triplet (F4, F2, F3) with separations of $\sim 13 \mu \mathrm{Hz}(12.7 \mu \mathrm{Hz}$ and $13.2 \mu \mathrm{Hz})$.

We found no viable model fits to all seven frequencies. To proceed, we split our analysis into two possible interpretations of the frequencies, that is, we assumed either F4, F2, and F3 are 


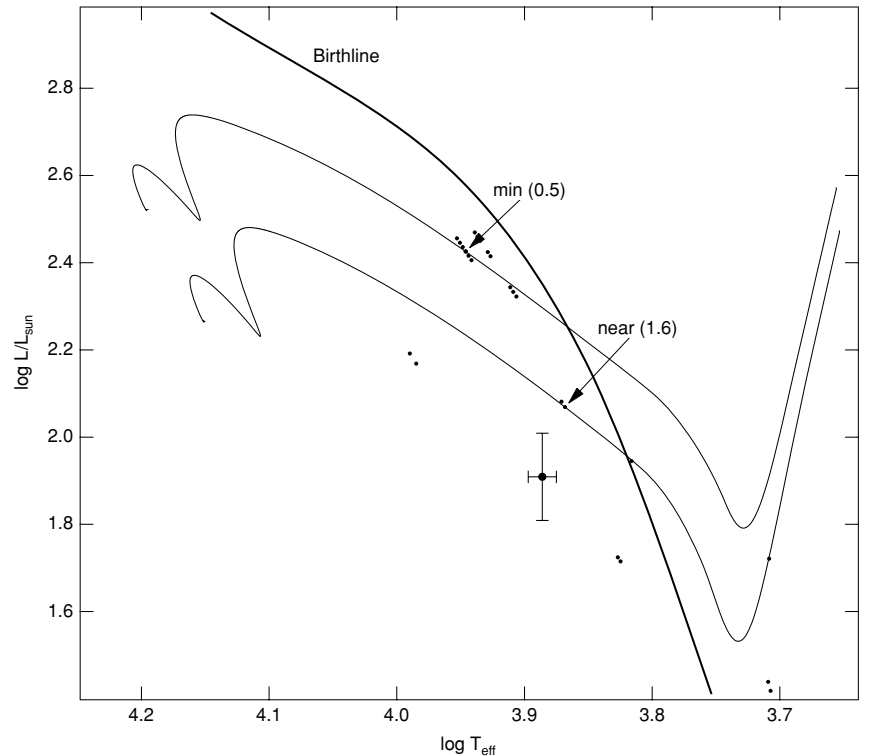

Figure 10. Theoretical HR diagram showing all models from our PMS grid whose $p$-mode oscillation spectra match the selected oscillation frequencies, F1, F2, F3, and F4 (i.e., the four largest amplitude modes) of V1 with $\chi^{2} \leqslant 2$. PMS tracks are drawn through the min and near models.

a rotational triplet or they are not. If they are then the frequency set becomes F1, F2, F5, F6, and F7 and if not, we retain the original sequence of seven frequencies.

We began our search for model fits by first considering only the three largest amplitude modes F1, F2, and F3, assuming no rotation, and then $\mathrm{F} 1, \mathrm{~F} 2$, and F5, assuming rotation. We found reasonable fits in both cases. We then added, one-by-one, lower amplitude frequencies until our model fits failed. We were unable to fit more than four frequencies at a time. Of course, under the assumption of rotation, fitting three frequencies accounts for five of the seven observed frequencies.

In Figure 10, we show the location of V1 (assuming it is a member of NGC 2264) along with all the model fits, with $\chi^{2}$ $\leqslant 2$, to frequencies $F 1, F 2, F 3$, and F4, i.e., to the four largest amplitude modes assuming no rotational splitting. Two models are singled out: near rot (see Table 2) is the model closest to the $T_{\text {eff }}$ (we are ignoring the luminosity) of $\mathrm{V} 1$ with $\chi^{2}=1.6$ and min rot is the model with the minimum $\chi^{2}=0.5$. Note, the $\chi^{2}$ are determined from fitting only the four largest amplitude modes.

The corresponding echelle diagrams for the min model is shown in Figure 11. The min mode comes close to fitting all but one of the model $p$-modes but, of course, the $T_{\text {eff }}$ of the model is far from the observed value.

Next we assume that $(\mathrm{F} 2, \mathrm{~F} 3, \mathrm{~F} 4)$ is a rotationally split $l=1$ $p$-mode centered on $\mathrm{F} 2$ and consider fits to the three frequencies F1, F2, and F5. We could not find viable model fits to F1, F2, F5, and F6, but could find to F1, F2, F5, and F7. There exist many model solutions scattered throughout the HR diagram. The model, labeled near rot, (see Table 2) that lies the closest to V1's position in the HR diagram has $\chi^{2}=1.1$, and the model labeled $\min$ no rot, lies far outside the $T_{\text {eff }}$ range for V1 but has the lowest $\chi^{2}$ value, 0.05 , of any of the models. The $\chi^{2}$ for the fits assuming rotational splittings are lower than those that do not.

If $\mathrm{V} 1$ is a field star then it is possible that it is a post-mainsequence star. We repeated the above analysis but searched for fits to the observed frequencies using our post-main-sequence

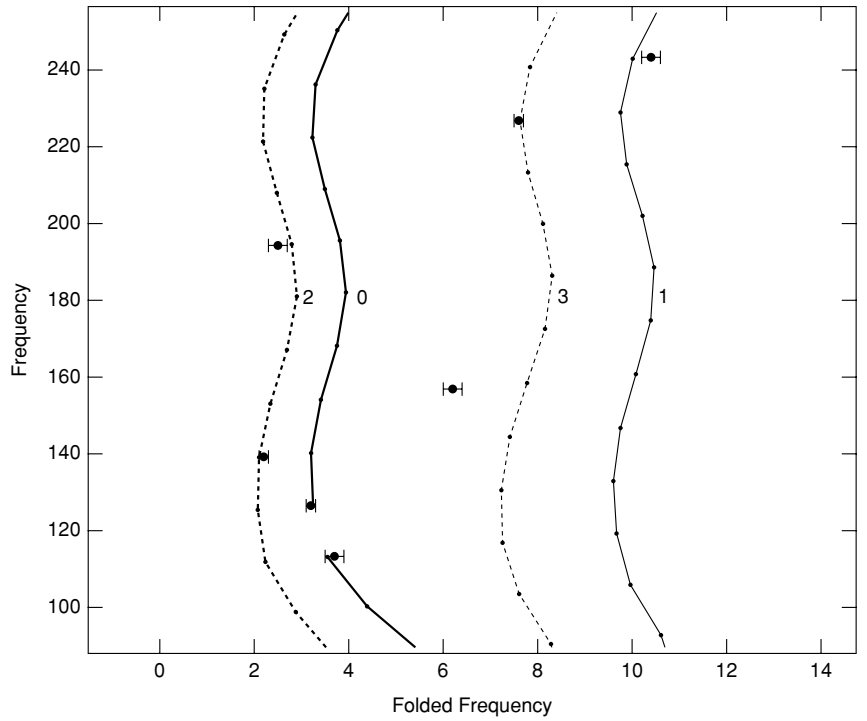

Figure 11. Echelle diagram (frequency vs. frequency modulo $13.7 \mu \mathrm{Hz}$ ) showing the seven observed frequencies of V1 along with the $p$-mode oscillation frequencies for the min model that fits the four largest amplitude frequencies $\left(\chi^{2}=0.5\right)$. Labels denote the angular degree of the model modes.

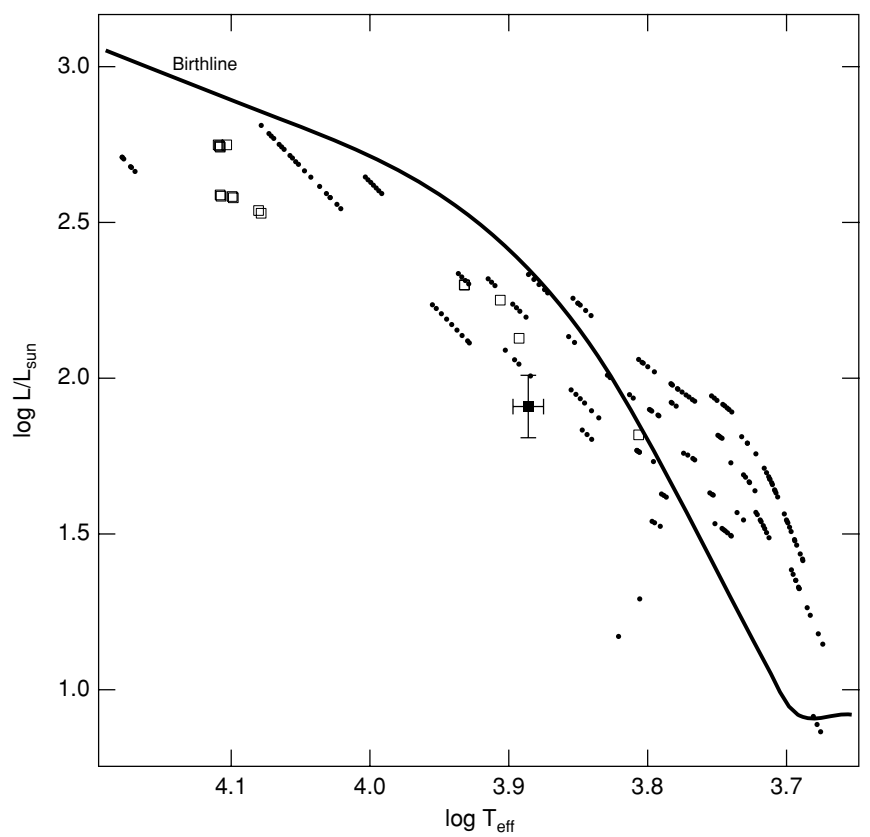

Figure 12. Theoretical HR diagram showing all models from our PMS grid (filled circles) and post-main-sequence grid (open squares) whose $p$-mode oscillation spectra match the two selected oscillation frequencies, F1 and F2 with $\chi^{2} \leqslant 2$.

grid (solar composition). No viable model fits were found with $\chi^{2} \leqslant 2$ for more than two frequencies. Figure 12 shows the model fits with $\chi^{2} \leqslant 2$ to the first two frequencies only. The post-main-sequence model fits, even for only two frequencies, are already sparse.

Finally, we examine in more detail the possibility that the triplet (F4, F2, F3) is a rotational splitting. The splitting is large, and this implies a high rotation rate. In Figure 13, we show a contour plot of the equatorial rotational velocity computed from the splitting normalized by the breakup velocity (the velocity at the surface of the star at the equator where the centrifugal acceleration equals the gravitational acceleration) as a function 


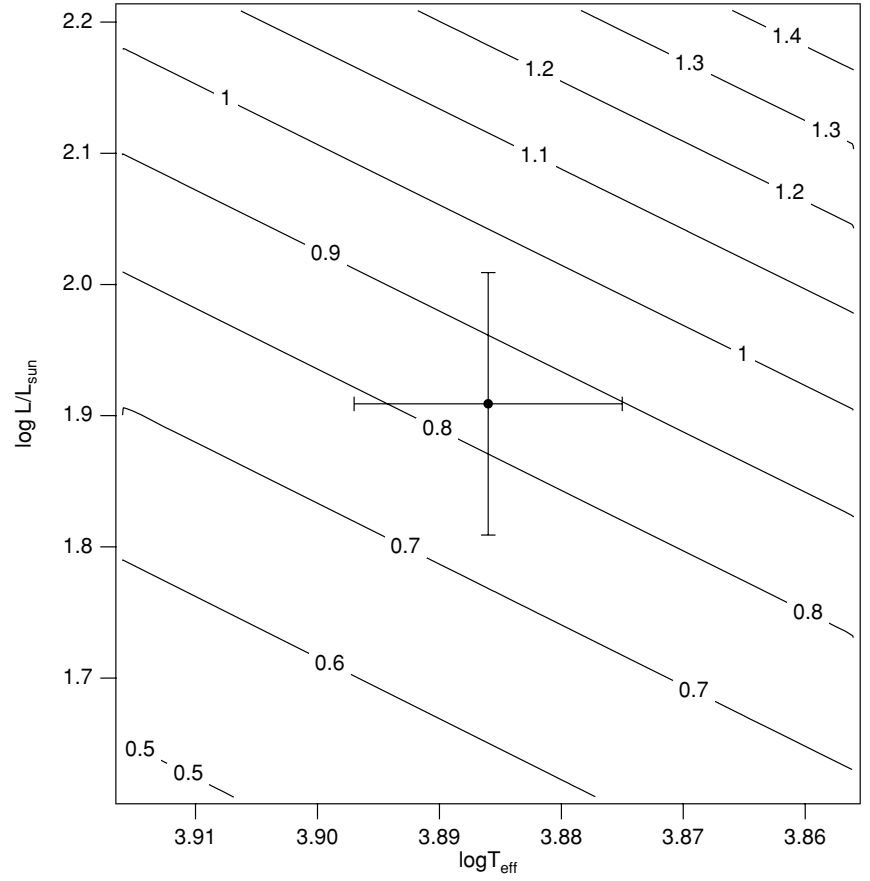

Figure 13. The rotational velocity in units of breakup velocity in the region of V1's position in the HR diagram (assuming it is a member of NGC 2264).

of $\log T_{\text {eff }}$ and $\log L / L_{\odot}$. The location of V1 is indicated by the point with error bars.

The absolute rotation velocity near V1 is approximately $290 \pm 30 \mathrm{~km} \mathrm{~s}^{-1}$. If the triplet is a rotational splitting then V1 is rapidly rotating, almost at breakup. As a consequence, its oscillation spectrum should show nonlinear effects due to rotation, i.e., asymmetric splittings.

For reference, in Table 2, we list some of the properties of the best-fitting models. We note that all model fits have masses $>3.0 M_{\odot}$ and have relatively young ages.

In summary, we have tried to confirm or refute V1's association with NGC 2264. We are not able to find fits to all of the observed frequencies, although we are able to find model fits to the largest amplitude frequencies. We also note that postmain-sequence model spectra provide an even poorer fit to the observed frequencies, suggesting that the star is indeed a PMS star.

\section{DISCUSSION}

By way of our modeling efforts, we see that the PMS pulsating stars that are believed to be members of NGC 2264 (V2, V3, V4, V588 Mon, and V589 Mon) divide into two groups. For convenience, we label them Group A, consisting of stars V2, V3, and V4, and Group B, consisting of stars V588 Mon and V589 Mon. Observationally, Group A stars have lower luminosities than Group B stars. For the stars considered here, Group A stars have fewer observed oscillation frequencies than Group B stars, but this may be due to their lower brightness, hence, lower $\mathrm{S} / \mathrm{N}$. With regard to modeling the stars, we find reasonably good PMS model fits, with $\chi^{2} \leqslant 1$, to the oscillation frequencies observed by MOST for V2, V3, and V4 and at the same time match their observed position in the HR diagram. The ages (both birthline age and classical age) of the models as implied by the seismic data are consistent with the age derived from other sources for NGC 2264. All Group A stars fall near the $2 M_{\odot}$
PMS evolutionary track. Along the track they all lie close to the ZAMS. In contrast, the oscillation spectra of Group B stars are not convincingly interpreted by the $p$-mode oscillation spectra of our classical PMS models. Because the oscillation frequencies of the two Group B stars are found in three distinct data sets and also in the space-based CoRoT observations (to be shown in a future paper), the frequencies are likely to be true intrinsic modes of the stars. Based on the location of the two Group B stars, V588 Mon and V589 Mon, in the HR diagram, as deduced from photometric data and the published distance to NGC 2264 and compared to our PMS evolutionary tracks, we estimate the mass of the stars to be $\sim 3 M_{\odot}$. The stars are located early on along their PMS evolutionary tracks, only recently dropping below the birthline.

The current evidence for V1 suggests that it is a PMS star since significantly fewer post-main-sequence models were found to fit the two largest amplitude modes compared to PMS model fits. If true then the star is likely to be a member of NGC 2264. The star's high proper motion could be explained if it were a binary with an invisible companion. Under these assumptions, then, V1 falls into Group B.

We initially believed that the reason behind Group B stars have poorer model fits than Group A stars is that Group B stars have more frequencies to fit. To test this hypothesis, we tried to fit only the two largest amplitude modes for each star in Group B. In both cases, we were unable to find any low $\chi^{2}$ model fits lying close to the stars' HR diagram locations. In other words, even when trying to fit two to four modes, similar to the number identified in V2, V3, and V4, we were still unable to obtain good model fits. This fact indicates that our success in PMS model fitting for the lower luminosity stars is not due to the lower number of available frequencies. Limiting the PMS model fitting to the same number of frequencies as in Group A stars for the more luminous Group B stars does not produce fits of comparable quality.

Reviewing the echelle diagrams for V589 Mon (Figure 9), one can see that although the model frequencies do not match very well the observed frequencies, the model frequency spacings show general agreement. V5887 Mon is similar but less pronounced. This suggests to us that the basic structure of our models is sound but that there exist perturbations to the structure that lie outside our model parameters.

Specifically, we suspect that either the surface structure of Group B stars is distinct from that predicted by our classical PMS models or that differential rotation within the star is splitting the mode frequencies. As stressed in the introduction, our PMS models start out on the Hayashi track where they are fully convective, hence, chemically homogeneous. Our models do not follow the effects of mass accretion, which not only increases the mass of the star as it adds material in layers to the surface, but also transfers angular momentum to the surface of the star from the surrounding accretion disk.

Stars with masses below $\sim 2.5 M_{\odot}$ emerge below the stellar birthline while still fully convective, whereas stars above this mass range emerge after the Hayashi phase with only surface convection (see Figure 1). For lower mass PMS stars, the effects of accretion on the structure are rapidly washed out by the total convection that occurs during the subsequent Hayashi phase (Palla \& Stahler). Consequently, the classical PMS models that we use are sufficient to model stars in this region of the HR diagram, i.e., Group A stars. Because the more massive PMS stars, i.e., Group B stars, do not enter a fully convective phase after accretion stops, the full effects of accretion on the structure 
of the star remain after the star falls below the birthline. Here for the first time, we believe, we may be seeing asteroseismic evidence of this.

Rotation, which we do not model here, may also account for our inability to fit the oscillation spectra of V588 Mon and V589 Mon. Here, though we note that if rotation is responsible in part for our modeling failures then somehow one has to explain the differences in the observed splittings among the multiplets (assuming that we have correctly identified them as multiplets) and their relation to the observed $v \sin i$. If large gradients in rotation exist in the interior then frequency-dependent rotational splittings could exist. In the case of V588 Mon, if either of the two closely spaced pairs of frequencies is due to solid body rotation then the inferred surface velocity at the equator is either 38 or $14 \mathrm{~km} \mathrm{~s}^{-1}$. This is incompatible with the observed $v \sin i=130 \mathrm{~km} \mathrm{~s}^{-1}$, and in the case of V589 Mon, if the triplet is interpreted as a rotationally split $l=1$ mode, then this implies an equatorial rotational velocity of $54 \mathrm{~km} \mathrm{~s}^{-1}$, which is consistent with the observed $v \sin i=60 \pm 10 \mathrm{~km} \mathrm{~s}^{-1}$. But the other two doublets are more closely spaced, hence, imply a slower rotation rate than would be consistent with $v \sin i$.

\section{SUMMARY AND CONCLUSIONS}

By analyzing the oscillation frequencies of five PMS stars from a single cluster, NGC 2264, we have shown that the oscillation spectra of classical PMS models can successfully fit the observed oscillation frequencies of the lower luminosity stars, i.e., the stars with masses $\sim 2 M_{\odot}$. Indeed, in the case of $\mathrm{V} 3$, with four observed frequencies we are able to locate two possible models out of a grid of $\sim 400,000$ models. On the other hand, our classical PMS models are unable to fit well the frequencies observed in the more massive (or luminous) PMS stars. We suggest that the reason for this distinction is primarily due to whether or not the star previously went through a Hayashi phase. The more massive PMS stars that do not go through a Hayashi phase retain some of the structural perturbations caused by mass accretion. Lower mass PMS stars that travel down the Hayashi track after falling below the birthline are thoroughly mixed, hence, nearly identical in structure to that predicted by classical PMS models.

The PMS models of Palla \& Stahler, which unlike ours follow the mass accretion during the protostar stage, could show distinct oscillation spectra from the classical models. The higher mass stars then should show the layering effects of mass accretion and deuterium burning. The mass that separates these two extremes depends on the location of the birthline and the modeling physics itself but is approximately $2.5 M_{\odot}$. An obvious next step for us is to compare the seismic modes of Palla \& Stahler-type PMS models to the observations.

The two distinct groups of PMS stars further distinguish themselves by the number of observed frequencies. The lower mass stars have far fewer observed frequencies than the higher mass stars. This is possibly an observational selection effect, since the lower mass stars are also less bright. Because we have restricted our analysis to adiabatic $p$-mode frequencies, we cannot comment on the driving and damping of specific modes. In a future work, we will look at the nonadiabatic characteristics of the model frequencies in order to address the apparent distinction in the number of frequencies observed between the two groups of models.

We also studied V1, a pulsating star located in the same region of the sky as the other stars, but with a distinct space motion. We were unable to draw any definitive conclusions about the nature of the star unless we assume it is a PMS star. If it is a PMS, as hinted at by our PMS model fit versus post-main-sequence model fit comparisons, then it is likely to be a member of NGC 2264 with a mass near $3 M_{\odot}$.

The Natural Sciences and Engineering Research Council of Canada supports the research of D.B.G., J.M.M., A.F.J.M., and S.M.R. A.F.J.M. is also supported by FQRNT (Québec). T.K., K.Z., and W.W.W. are supported by the Austrian Research Promotion Agency (FFG) and the Austrian Science Fund (FWF; T.K. and W.W.W. through project P17580; KZ through project T 335-N16).

\section{REFERENCES}

Alexander, D. R., \& Ferguson, J. W. 1994, ApJ, 437, 879

Baglin, A., Auvergne, M., Barge, P., Buey, J.-T., Catala, C., Michel, E., Weiss, W., \& the COROT Team 2002, in Proc. 1st Eddington Workshop on Stellar Structure and Habitable Planet Finding, 11-15 June 2001, Córdoba, Spain ed.: B. Battrick; scientific ed.: F. Favata, I. W. Roxburgh, \& D. Galadi (ESA SP-485; Noordwijk: ESA Publications Division), 17

Bahcall, J. N., Pinsonneault, M. H., \& Basu, S. 2001, ApJ, 555, 990

Böhm-Vitense, E. 1958, Z. Astrophys., 46, 108

Chandrasekhar, S. 1957, An Introduction to the Study of Stellar Structure (New York: Dover)

D’Antona, F., \& Mazzitelli, I. 1994, ApJS, 90, 467

Demarque, P., Guenther, D. B., Li, L. H., Maxumdar, A., \& Straka, W. S. 2008, Ap\&SS, 316, 31

Guenther, D. B. 1994, ApJ, 422, 400

Guenther, D. B., \& Brown, K. I. T. 2004, ApJ, 600, 419

Guenther, D. B., Kallinger, T., Zwintz, K., Weiss, W. W., \& Tanner, J. 2007, ApJ, 671,581

Guenther, D. B., et al. 2005, ApJ, 635, 547

Hayashi, C. 1961, PASJ, 13, 450

Hog, E., et al. 1998, A\&A, 335, 65

Iben, I. 1965, ApJ, 464, 943

Iglesias, C. A., \& Rogers, F. J. 1996, ApJ, 464, 943

Kallinger, T., Zwintz, K., \& Weiss, W. 2008, A\&A, 488, 279

Kharchenko, N. V. 2001, Kinematics and Physics of Celestial Bodies, 17, 409

Lane, J. H. 1869, Am. J. Sci., 50, 452 (2nd Ser.)

Larson, R. B. 1972, MNRAS, 157, L121

Palla, F., \& Stahler, S. W. 1992, ApJ, 392, 667

Palla, F., \& Stahler, S. W. 1993, ApJ, 418, 414

Palla, F., \& Stahler, S. W. 1999, ApJ, 525, 772

Pribulla, T., Rucinski, S. M., Kuschnig, R., Ogloza, W., \& Pilecki, B. 2009, MNRAS, 392, 847

Rogers, F. J. 1986, ApJ, 310, 723

Rogers, F. J., Swenson, F. J., \& Iglesias, C. A. 1996, ApJ, 456, 902

Sagar, R., Piskunov, A. E., Miakutin, V. I., \& Joshi, U. C. 1986, MNRAS, 220, 383

Stahler, S. W. 1983, ApJ, 274, 822

Stahler, S. W., Shu, F. H., \& Taam, R. E. 1980a, ApJ, 241, 637

Stahler, S. W., Shu, F. H., \& Taam, R. E. 1980b, ApJ, 242, 226

Stahler, S. W., Shu, F. H., \& Taam, R. E. 1981, ApJ, 248, 727

Tassoul, M. 1980, ApJS, 43, 469

Walker, G. A. H., et al. 2003, PASP, 115, 1023

Walker, M. F. 1956, ApJS, 2, 365

Yi, S. K., Kim, Y.-C., \& Demarque, P. 2003, ApJS, 144, 259

Zwintz, K., \& Weiss, W. W. 2006, A\&A, 457, 237

Zwintz, K., Guenther, D. B., \& Weiss, W. W. 2007, ApJ, 655, 342

Zwintz, K. 2008, ApJ, 673, 1088

Zwintz, K., et al. 2009, A\&A, 502, 239 